\title{
Detector-free photothermal bar-chart microfluidic chips (PT-Chips) for visual quantitative detection of biomarkers
}

\author{
Wan Zhou, ${ }^{1+}$ Guanglei Fu, ${ }^{1,2+}$ and Xiujun $\mathrm{Li}^{1,3,4^{*}}$ \\ ${ }^{1}$ Department of Chemistry and Biochemistry, The University of Texas at El Paso, 500 West \\ University Ave, El Paso, TX 79968, USA \\ ${ }^{2}$ Biomedical Engineering Research Center, Medical School of Ningbo University, Ningbo, \\ Zhejiang, 315211, P. R. China \\ ${ }^{3}$ Border Biomedical Research Center, Biomedical Engineering, The University of Texas at El \\ Paso, 500 West University Ave, El Paso, TX 79968, USA \\ ${ }^{4}$ Environmental Science and Engineering, The University of Texas at El Paso, 500 West \\ University Ave, El Paso, TX 79968, USA \\ ${ }^{+}$Denotes equal contributions. \\ *Corresponding Author: XiuJun Li; Email: xli4@utep.edu
}

\begin{abstract}
$\underline{\text { Abstract }}$
The volumetric bar-chart microfluidic chips (V-Chips) driven by chemical reaction-generated gas provide a promising platform for point-of-care (POC) visual biomarker quantitation. However, multiple limitations are encountered in conventional V-Chips, such as costly and complex chip fabrication, complicated assembly, and imprecise controllability of gas production. Herein, we introduced nanomaterial-mediated photothermal effects to V-Chips, and for the first time developed a new type of V-Chip, photothermal bar-chart microfluidic chip (PT-Chip), for visual quantitative detection of biochemicals without any bulky and costly analytical instruments. Immunosensing signals were converted to visual readout signals via photothermal effects, the onchip bar-chart movements, enabling quantitative biomarker detection on a low-cost polymer hybrid PT-Chip with on-chip scale rulers. Four different human serum samples containing prostate-specific antigen (PSA) as a model analyte were detected simultaneously using the PTChip, with the limit of detection of $2.1 \mathrm{ng} / \mathrm{mL}$, meeting clinical diagnostic requirements. Although no conventional signal detectors were used, it achieved comparable detection sensitivity to absorbance measurements with a microplate reader. The PT-Chip was further validated by testing
\end{abstract}


human whole blood without the color interference problem, demonstrating good analytical performance of our method even in complex matrixes and thus the potential to fill a gap in current clinical diagnostics that is incapable of testing whole blood. This new PT-Chip driven by nanomaterial-mediated photothermal effects opens a new horizon of microfluidic platforms for instrument-free diagnostics at the point of care.

Keywords: Photothermal bar-chart microfluidic chip; Point-of-care testing; Quantitative detection; Photothermal immunoassay; Biomarkers. 


\section{Introduction}

Protein biomarkers play an important role in disease screening, disease diagnosis, prognosis, treatment responses, and therapeutic decisions. ${ }^{1-3}$ Currently, various methods have been developed for the detection of protein biomarkers, including surface plasmon resonance (SPR), ${ }^{4}$ colorimetry, ${ }^{5,6}$ fluorescence, ${ }^{7}$ electrochemistry, ${ }^{8}$ and chemiluminescence. ${ }^{9}$ Unfortunately, these conventional methods still face great challenges for point-of-care (POC) application, especially in resource-limited settings. For instance, colorimetric detection is one of the most widely used methods in enzyme-linked immunosorbent assays (ELISAs), leveraging its simple and visual color changes; whereas the sensitivity and selectivity of colorimetric methods are always compromised..$^{10}$ Additionally, colorimetric methods are vulnerable to color interferences and can only provide qualitative or semi-quantitative data, unless a specialized instrument such as a spectrophotometer is used. Such issues also limit the development of near-patient whole blood testing, which are conducted as closely as possible after specimen collection and eliminate the centrifugation process required in current serological tests. ${ }^{11,}{ }^{12}$ Although some other detection methods (e.g., SPR, fluorescence, and electrochemistry) can provide high sensitivity and selectivity, ${ }^{13}$ they rely on expensive biochemicals (e.g., enzymes), costly and bulky instruments (e.g., fluorescence microscopy and microplate readers), and trained personnel. Therefore, there has been a sustained need for developing new immunosensing strategies for low-cost, simple, and portable POC detection of protein biomarkers.

Microfluidic lab-on-a-chip (LOC) technology offers a powerful diagnostic tool for the POC detection of various biomolecules, with various significant advantages such as low reagent consumption, fast assays, miniaturization, high degrees of integration, and portability. ${ }^{14-20}$ Recently, a novel POC microfluidic platform has been developed by Qin and co-workers, namely the volumetric bar-chart microfluidic chip (V-Chip), which shows great potential for visual biochemical quantitation. ${ }^{21}$ By applying catalase as the ELISA probe, oxygen gas was generated and accumulated within microfluidic channels, leading to increased pressure to push the preloaded ink to move through microfluidic channels in the V-Chip. Because the as-produced moving distances were correlated with the concentration of protein biomarkers, the V-Chip achieved quantitative measurements of analytes without using costly detectors. By combining different immunoassays with V-Chips, various detection platforms have been explored thereafter to detect a broad range of biomolecular targets, leveraging the convenient analytical readouts. ${ }^{21-27}$ Despite 
the promising potential of V-Chips in POC testing, there are multiple drawbacks hurdling their wide applications for POC detection and other applications. For example, the fabrication of glass microfluidic V-Chips is complicated, and requires stringent cleanroom facilities, resulting in high cost. Besides, to obtain good sealing of V-Chips, V-Chips are needed to be assembled and aligned in a specific configuration on site by trained personnel, making it challenging for biosensing at the point of care. More significantly, all current V-Chips are driven by gas generation via chemical/enzymatic reactions, which lack controllability of reactions once they start and stability due to inevitable denaturation of enzymes at the point of care. There is a high demand for the development of new types of V-Chips by introducing new types of robust driving forces.

Nanomaterial-mediated photothermal effects have attracted a lot of attention, due to the unique light-to-heat conversion property, invasiveness, and compatibility in many biological systems. ${ }^{28-}$ 33 Photothermal effects have been extensively studied in photothermal cancer therapy for more than two decades, but they have not been explored for biosensing until recently. ${ }^{6,34-41}$ For example, our group developed the first photothermal immunoassay for the detection of cancer biomarkers using a thermometer, avoiding the need for expensive analytical instruments. ${ }^{34}$ Most recently, by applying gold nanoparticle aggregates as photothermal probes, another quantitative biosensing platform was developed for genetic analysis based on the gold nanoparticle aggregation-induced photothermal effects. ${ }^{36}$ Only using a thermometer, target nucleic acids were quantified with high sensitivity and specificity, where the concentration of target nucleic acids was correlated with the temperature increase upon irradiation of a near-infrared (NIR) laser. ${ }^{36}$ These studies have motivated us to initiate new types of application of photothermal effects as new driving forces in bar-chart microfluidic chips.

In this work, we applied nanomaterial-mediated photothermal effects to microfluidic chips and developed a new type of V-Chips, photothermal bar-chart microfluidic chip (PT-Chip) driven by photothermal effects, for quantitative biochemical analysis without using any bulky and costly detectors. This photothermal platform was composed of inexpensive materials, poly (methyl methacrylate) (PMMA) and polydimethylsiloxane (PDMS), for the fabrication of hybrid photothermal microfluidic chips. By introducing the iron oxide nanoparticles $\left(\mathrm{Fe}_{3} \mathrm{O}_{4} \mathrm{NPs}\right)$ mediated photothermal effect into the volumetric bar-chart microchip, the produced PT-Chip was used for visual quantitative immunosensing of protein biomarkers, without the aid of any detectors. $\mathrm{Fe}_{3} \mathrm{O}_{4}$ NPs involved in a typical sandwich immunoassay were converted to Prussian blue 
nanoparticles (PB NPs), a strong NIR photothermal agent. PB NPs were then exploited to generate heat under the laser irradiation, acting as a powerful driving force in the PT-Chip. As such, the immunosensing signals were converted to visual bar charts on the PT-Chip. The quantitation of biomolecules was achieved by visually reading the colored bar-chart distance on the PT-Chip, without the aid of any bulky and expensive instruments. As a model target, a cancer biomarker, prostate-specific antigen (PSA), was spiked in different media (PBS buffer and normal human serum samples) and successfully detected using this PT-Chip. Visual quantitative measurements of PSA were achieved, with the limits of detection (LOD) of 2.0 and $2.1 \mathrm{ng} / \mathrm{mL}$ in the PBS buffer and serum samples, respectively. The sensitivity of the on-chip detection of PSA meets the clinical requirement of prostate cancer diagnostics. This method was further validated by testing spiked PSA in human whole blood samples without the issues of color interferences, with satisfactory analytical recoveries. This PT-Chip provides a new low-cost platform for quantitative POC analysis without using costly instruments, with ease of operations, minimal color interferences, and a new robust and controllable driving force.

\section{$\underline{\text { Experimental section }}$}

\section{Materials}

Chemicals and materials, including bovine serum albumin (BSA), human serum, Dulbecco's phosphate-buffered saline (PBS buffer, $10 \mathrm{mM}, \mathrm{pH}$ 7.4), and prostate-specific antigen (PSA), were purchased from Sigma (St. Louis, MO, US). Citric acid monohydrate and 1-Ethyl-3-[3dimethylaminopropyl] carbodiimide hydrochloride $(\mathrm{EDC} \cdot \mathrm{HCl})$ were purchased from VWR (Radnor, PA, US). Hydrogen peroxide $\left(\mathrm{H}_{2} \mathrm{O}_{2}, 30 \% \mathrm{w} / \mathrm{w}\right), \mathrm{N}$-hydroxysulfosuccinimide (SulfoNHS), and potassium ferrocyanide $\left(\mathrm{K}_{4}\left[\mathrm{Fe}(\mathrm{CN})_{6}\right]\right)$ were purchased from Fisher Scientific (Hampton, NH, US). Monoclonal mouse anti-human PSA antibody and polyclonal rabbit antihuman PSA antibody were obtained from Abcam (Cambridge, MA, US). Fresh human whole blood was purchased from Zen-Bio (Morrisville, NC, US) and has been tested by FDA licensed tests, showing negative results in relevant items. Iron oxide nanoparticles $\left(\mathrm{Fe}_{3} \mathrm{O}_{4} \mathrm{NPs}_{\mathrm{s}}\right.$ with carboxylic acid groups, $30 \mathrm{~nm}$ in diameter) were purchased from Ocean NanoTech (San Diego, CA, US). The silicone elastomer base and the curing agent (Sylgard 184) for the fabrication of polydimethylsiloxane (PDMS) were obtained from Dow Corning (Midland, MI, US). Poly (methyl methacrylate) (PMMA, $1.5 \mathrm{~mm}$ and $2.0 \mathrm{~mm}$ in thickness) was purchased from Mcmaster-Carr (Los Angeles, CA, US). Unless otherwise noted, all chemicals were used as received without 
further purification and Milli-Q water $(18.2 \mathrm{M} \Omega \cdot \mathrm{cm})$ was used throughout the study from a Millipore system (Bedford, MA, US). The $\mathrm{pH}$ values of all buffer solutions were determined using a pH meter purchased from Fisher Scientific (Model AB15, Hampton, NH, US).

\section{Fabrication of the PDMS/PMMA hybrid PT-Chip}

The PDMS/PMMA hybrid PT-Chip $(7.5 \mathrm{~cm} \times 7.5 \mathrm{~cm})$ consisted of three layers, including a top PDMS lid, a middle PDMS layer, and a bottom PMMA layer, as illustrated in the exploded view in Fig. 1. Both PDMS layers were fabricated according to a modified soft lithography procedure that we published previously. ${ }^{42}$ Typically, the liquid PDMS base and the curing agent were mixed thoroughly on a mechanical stirrer (IKA RW16, VWR, Radnor, PA, US) with a weight ratio of $8.5: 1$. The precursor mixture was poured into a petri dish and degassed in a vacuum desiccator for $0.5 \mathrm{~h}$ to remove air bubbles. The mixture was then incubated in an oven at $50{ }^{\circ} \mathrm{C}$ for $0.5 \mathrm{~h}$ and then peeled off from the petri dish. The obtained PDMS sheet $(2.0 \mathrm{~mm}$ in thickness) was cut into the designed shape and punched using biopsy punches, forming the inlet reservoirs and the outlet reservoirs with diameters of $0.35 \mathrm{~cm}$ and $0.40 \mathrm{~cm}$, respectively.

The patterned PMMA layer was designed with Adobe AI software as shown in Fig. 1A including 4 individual units for photothermal measurements only upon one-time laser irradiation. Each unit contains one microwell acting as both the sample inlet and the laser irradiation spot, one microchannel for sample flowing upon the laser irradiation, and one scale ruler for convenient signal reading. This layer was fabricated from a PMMA sheet (1.5 mm in thickness) using a laser cutter (Epilog laser, Golden, CO, US). The reservoirs were cut using the laser raster mode at the power of $50 \%$ and the speed of $25 \%$, with a diameter of $0.35 \mathrm{~cm}$ and a depth of $830 \mu \mathrm{m}$. The microchannels were laser ablated using the laser raster mode at the power of $40 \%$ and the speed of $30 \%$, with the total length of $50 \mathrm{~mm}$, the width of around $250 \mu \mathrm{m}$, and the depth of $300 \mu \mathrm{m}$. The on-chip scale rulers were customized with each unit equivalent to $2.0 \mathrm{~mm}$. The frame of the PMMA layer was cut using the laser vector mode at the power of 35\% and the speed of $30 \%$. When each layer is ready, the two PDMS layers and the PMMA layer were assembled reversibly based on the self-adhesion (Fig. 1B). ${ }^{43}$ Basically, the bottom PMMA layer was sealed first by aligning with the middle PDMS layer. Then, the immunosensing solution was introduced to the PT-Chip through the center inlets. Finally, the top PDMS layer was used to seal the inlets prior to the laser irradiation. 

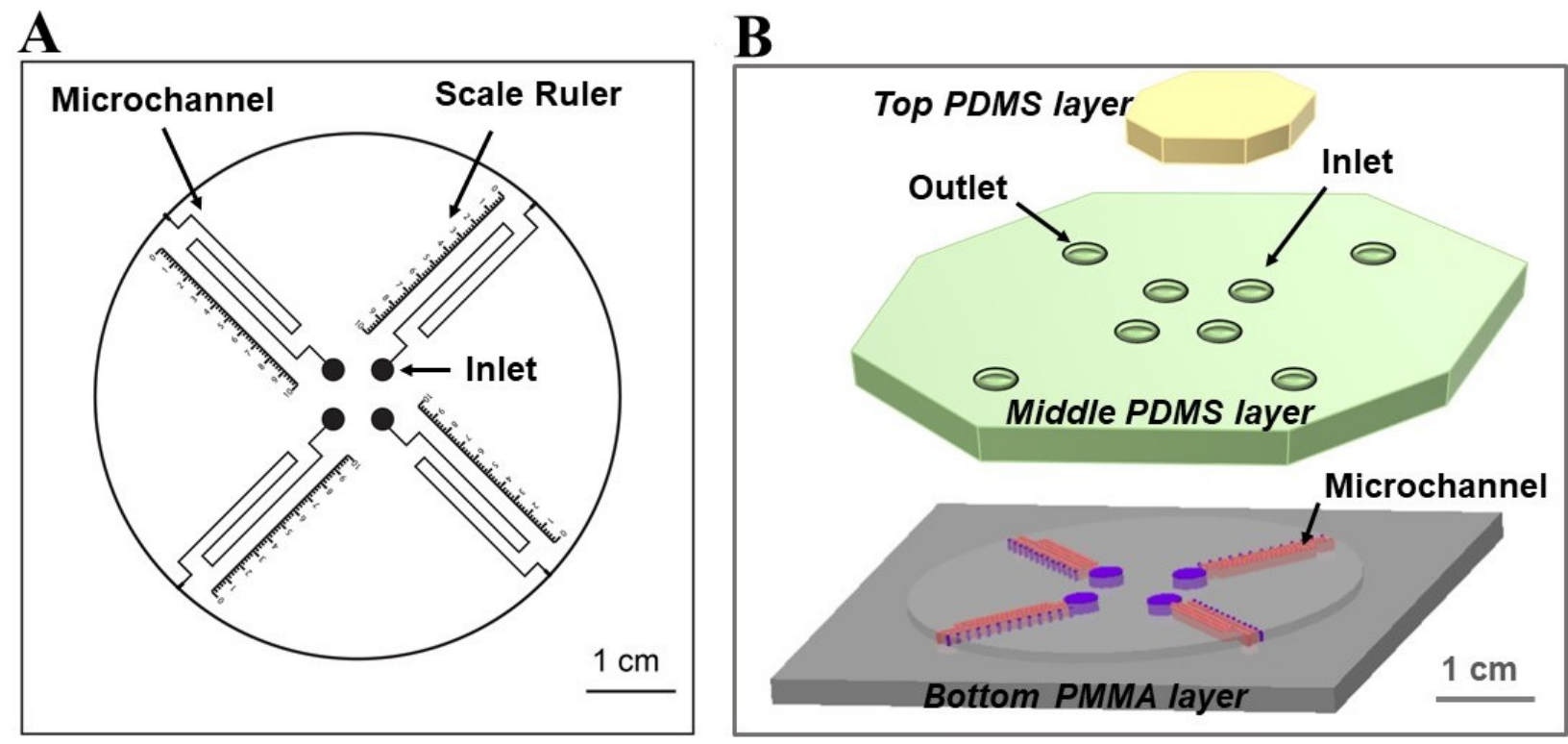

Fig. 1 The top view (A) and the exploded view (B) of the PDMS/PMMA hybrid PT-Chip.

\section{Preparation of the antibody conjugated $\mathrm{Fe}_{3} \mathrm{O}_{4} \mathrm{NPs}$}

The antibody-conjugated $\mathrm{Fe}_{3} \mathrm{O}_{4}$ NPs were synthesized via a typical carbodiimide crosslinking reaction modified from a previously published protocol. ${ }^{34}$ Particularly, $\mathrm{Fe}_{3} \mathrm{O}_{4} \mathrm{NPs}$ with carboxylic acid groups were dispersed in water to achieve the final concentration of $0.25 \mathrm{mg} / \mathrm{mL}$. A $25 \mu \mathrm{L}$ mixture containing $25.0 \mathrm{mg} / \mathrm{mL}$ of $\mathrm{EDC} \cdot \mathrm{HCl}$ and $29.8 \mathrm{mg} / \mathrm{mL}$ of Sulfo-NHS was added into the above nanoparticle dispersion and allowed to react for $40 \mathrm{~min}$ at room temperature under gentle shaking. Then, $40 \mu \mathrm{g}$ polyclonal rabbit anti-human PSA antibody was added into the nanoparticle dispersion, followed by adjusting the $\mathrm{pH}$ of the mixture to 8.0. After further incubation for $2.0 \mathrm{~h}$ at room temperature under gentle shaking, the antibody conjugated $\mathrm{Fe}_{3} \mathrm{O}_{4} \mathrm{NPs}$ were collected via the centrifugation of the above mixture at 11,000 rpm for $7 \mathrm{~min}$. The pellet was then washed three times with PBS buffer $(\mathrm{pH} 7.4,10 \mathrm{mM})$. Finally, the antibody conjugated $\mathrm{Fe}_{3} \mathrm{O}_{4} \mathrm{NPs}_{\text {s }}$ were dispersed in $1.0 \mathrm{~mL}$ PBS buffer ( $\mathrm{pH} 7.4,10 \mathrm{mM}$, containing $0.2 \% \mathrm{BSA}$ ), and stored at $4{ }^{\circ} \mathrm{C}$ prior to use.

\section{Nanomaterial-mediated immunosensing procedures}

$100 \mu \mathrm{L}$ of $30 \mu \mathrm{g} / \mathrm{mL}$ monoclonal mouse anti-human PSA antibody was incubated overnight at $4.0{ }^{\circ} \mathrm{C}$ in a PCR tube. Then, $200 \mu \mathrm{L}$ of $5 \%$ BSA blocking buffer was added to block the unbinding 
sites for $0.5 \mathrm{~h}$ at $37^{\circ} \mathrm{C}$. Different standard PSA solutions (100 $\left.\mu \mathrm{L}\right)$ were prepared in PBS buffer containing $0.5 \% \mathrm{BSA}$ and incubated in the above tube for $2.0 \mathrm{~h}$ at $37^{\circ} \mathrm{C}$, followed by thoroughly washing with a PBS buffer. The polyclonal anti-human PSA antibody conjugated $\mathrm{Fe}_{3} \mathrm{O}_{4} \mathrm{NPs}_{\mathbf{s}}(100$ $\mu \mathrm{L}$ ) were added and allowed for another incubation for $2.0 \mathrm{~h}$ at $37^{\circ} \mathrm{C}$. The tube carrying sandwich immunosensing samples was finally washed with a PBS buffer thoroughly.

To validate the photothermal bar-chart chip, different concentrations of PSA were spiked in 5-fold diluted normal human serum samples and 10-fold diluted fresh human whole blood samples, respectively. It was noted that diluted normal human samples were used in these tests to avoid any unspecific binding from heterophilic antibodies in real samples (e.g., serum and whole blood samples) in the immunoassays. ${ }^{44}$ To test the selectivity of our method, three kinds of interfering substances, containing hepatitis B surface antigen ( $\mathrm{HBsAg}$ ), carcinoembryonic antigen (CEA), and immunoglobulin $\mathrm{G}(\operatorname{IgG})$ at 10 -fold higher concentrations than PSA (up to $80 \mathrm{ng} / \mathrm{mL}$ ) were used for the on-chip specificity testing, which were also prepared in these diluted normal human serum samples and fresh human blood samples, respectively.

\section{Nanoparticles transformation procedures}

After completing the aforementioned immunocomplexing reactions, iron oxide nanoparticles were converted to a stronger photothermal probe, Prussian blue nanoparticles. A $120 \mu \mathrm{L} 0.1 \mathrm{M} \mathrm{HCl}$ solution was added to the above tube carrying sandwich immunosensing samples, followed by the ultrasonication for $40 \mathrm{~min}$ at room temperature. Then, a $30 \mu \mathrm{L}$ of $90.0 \mathrm{mM} \mathrm{K}_{4}\left[\mathrm{Fe}(\mathrm{CN})_{6}\right]$ aqueous solution was used to facilitate the reaction between ferric ions and ferrocyanide ions under acidic conditions, in which spherical $\mathrm{Fe}_{3} \mathrm{O}_{4} \mathrm{NPs}$ captured in the sandwich immunoassay were effectively transformed to PB NPs with cubic nanostructures. ${ }^{45}$ The nanoparticle transformation reaction was depicted in Fig. S1. During the reaction, the immunosensing solution was mixed intensively every $10 \mathrm{~min}$, and allowed to react for $1.0 \mathrm{~h}$ at room temperature. The obtained immunosensing solutions were characterized using UV-vis spectra via a microplate reader (Molecular Devices, Sunnyvale, CA, US) prior to on-chip detection.

\section{Visual quantitative detection of PSA on the PDMS/PMMA hybrid PT-Chip}

The visual quantitative detection of PSA based on nanoparticles-mediated photothermal effect was performed under the irradiation of an $808 \mathrm{~nm}$ diode laser with a small footprint (Opto Engine, 
Midvale, UT, US. $5 \times 2.7 \times 1.7^{\prime \prime}$ ). Four microliters of food dye solution were added into the above immunosensing solution for enhanced visualization of fluid movements. Each inlet reservoir on the PT-chip was loaded with $30 \mu \mathrm{L}$ sample solution and then sealed with the top PDMS layer. Four individual reservoirs containing sample solutions were exposed to the NIR laser at the power density of $2.21 \mathrm{~W} / \mathrm{cm}^{2}$ simultaneously. The increased vapor pressure originated from the photothermal reagent (PB NPs) would force fluids to flow through the microchannels. Upon laser

irradiation for $5 \mathrm{~min}$, the moving distances of colored fluids were observed and recorded directly as bar charts on the PT-Chip. Visual quantitative detection of target PSA was achieved by reading the on-chip moving distance $(\Delta \mathrm{L})$ from its corresponding on-chip scale ruler, which was derived from the new driving force provided by nanomaterial-mediated photothermal effects.

\section{$\underline{\text { Results and Discussion }}$}

\section{Working principle of the PT-Chip}

The nanomaterial-mediated photothermal effect was introduced to the PDMS/PMMA hybrid PTChip for the visual quantitative detection of biomarkers. The working principle of the PT-Chip is illustrated in Scheme 1, in which a typical sandwich-type ELISA is applied as a proof of concept, where the monoclonal antibody is immobilized on the PCR tube surface acting as the capture antibody. The prostate cancer biomarker PSA is used as the model target antigen, specifically binding with the capture antibody as well as the $\mathrm{Fe}_{3} \mathrm{O}_{4}$ NPs-labeled polyclonal antibody via immune recognition interactions. In the presence of the target $\mathrm{PSA}, \mathrm{Fe}_{3} \mathrm{O}_{4} \mathrm{NPs}$ are introduced and immobilized on the PCR tube surface via the sandwich structure immunoassay. The photothermal effect of $\mathrm{Fe}_{3} \mathrm{O}_{4} \mathrm{NPs}$ was found to be too weak to generate strong photothermal biosensing signals, ${ }^{46}$ and thus $\mathrm{Fe}_{3} \mathrm{O}_{4}$ NPs are converted into a strong NIR photothermal reagent, PB NPs, due to the strong absorption in the NIR region via a simple complexation reaction under acidic conditions (Fig. S1). Since the amount of captured $\mathrm{Fe}_{3} \mathrm{O}_{4} \mathrm{NPs}$ is related to the amount of the target, the converted PB NPs will be thus correlated with the concentration of the target. After the conversion and the addition of a food dye to enhance the visual effect of the flow movement, the as-prepared solution will be loaded into a microwell and sealed in the PT Chip. When the microwell is irradiated with a NIR laser, the conversion from NIR light to heat is triggered via the nanomaterialmediated photothermal effect. The heat is accumulated continuously and causes a dramatic increase in vapor pressure in the microwell of the PT-Chip, in which the pressure is transduced to 
drive colored sample solutions to move into the connected microchannel. It is worth noting that an on-chip scale ruler (with each unit equivalent to $2.0 \mathrm{~mm}$ ) is designed for the convenience of observation and recording of the moving distance. Because the generated heat is related to the amount of PB NPs, the moving distance of the colored solution will be correlated with the original target concentration, thus enabling visual quantitative detection of PSA by using the bar chart height as detection signals via reading the on-chip scale ruler on the PT-Chip, without using other costly signal detectors. As such, the immunosensing signals are converted to visual quantitative readouts (i.e., on-chip moving distances) using the PT-Chip. In the absence of the target analyte, no specific binding between PSA and capture antibodies will occur. Hence, no $\mathrm{Fe}_{3} \mathrm{O}_{4}$ NPs will be captured or converted to PB NPs, resulting in no noticeable bar-chat movement in microfluidic channels.

Moreover, as shown in Fig. 1 and Scheme 1, four separate units are designed in a radial pattern from the center of the PT-Chip. This radial multi-channel pattern enables us to perform four individual experiments at the same time with only one-time laser irradiation. Thus, visual quantitative analysis of different targets, different concentrations of one target, or four different replicates of the same sample can be achieved simultaneously from one-time laser irradiation. Although only four channels were designed in this work to demonstrate the proof of the concept, they can be scaled up for higher-throughput visual quantitative analysis without using external detectors. 


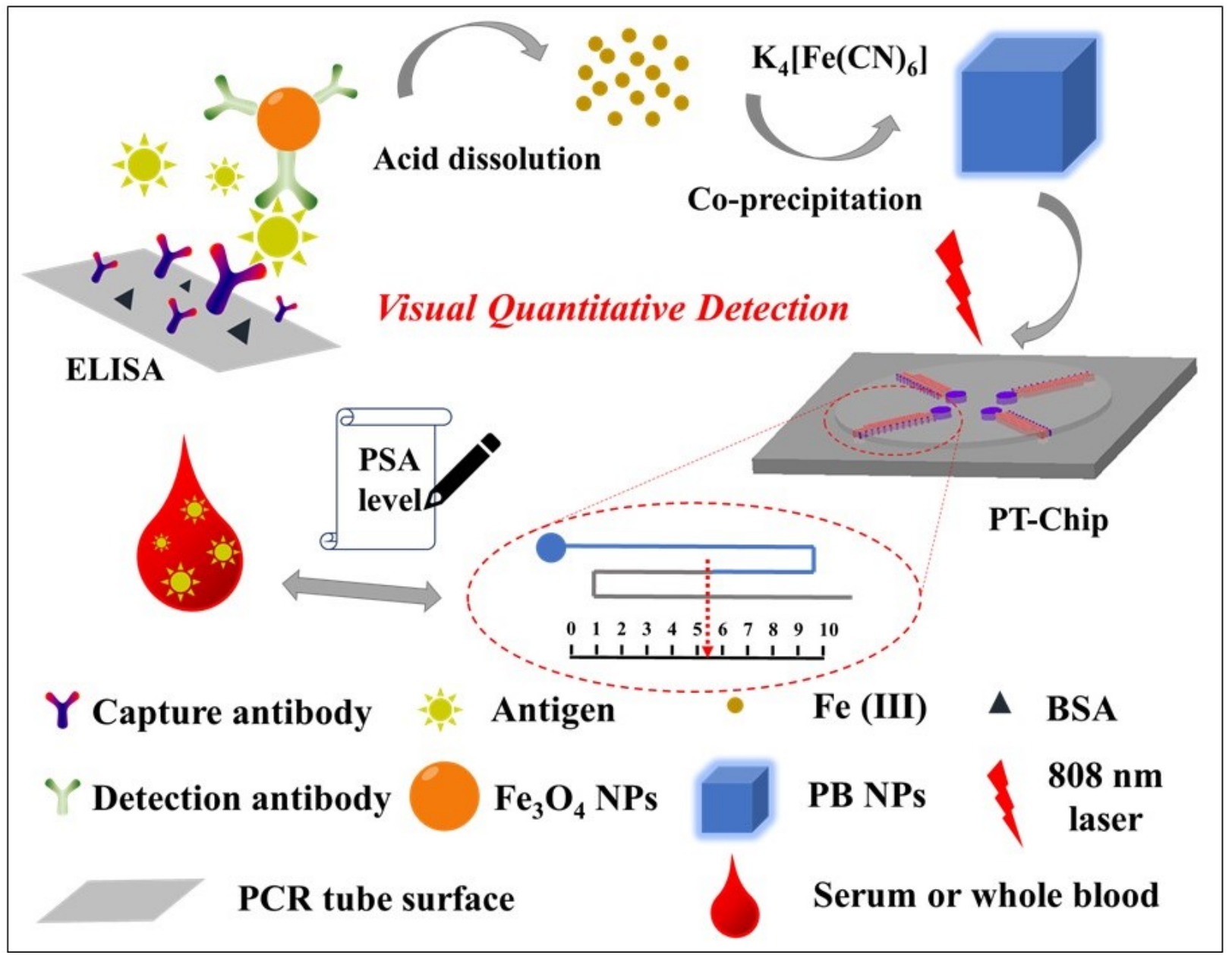

Scheme 1 Working principle of the visual quantitative detection of PSA based on the nanomaterial-mediated photothermal bar-chart microfluidic chip.

\section{UV-vis characterization of PSA detection in PBS}

To investigate the nanomaterial-mediated immunosensing process, UV-Vis spectroscopic characterization was carried out in a 96-well microplate using a microplate reader. Fig. 2 shows the color changes and UV-vis spectra at different concentrations of standard PSA from 0 to 64.0 $\mathrm{ng} / \mathrm{mL}$, which were prepared in a PBS buffer $(\mathrm{pH} \mathrm{7.4,} 10 \mathrm{mM})$. After the transformation of nanoparticles, it was clearly observed that the color changed from colorless or light yellow to blue (i.e., Prussian blue), when increasing the concentration of PSA in the range from 0 to $64.0 \mathrm{ng} / \mathrm{mL}$. The spectra in Fig. 2A exhibited stronger absorbance in the NIR range with the concentration increase of the target, implying the transformation of more $\mathrm{Fe}_{3} \mathrm{O}_{4}$ NPs into PB NPs and stronger photothermal effects. The peaks at $748 \mathrm{~nm}$ proved to be the representative maximum absorbance 
of PB NPs, which was consistent with the previous literature. ${ }^{45}$ Hence, the color changes and spectra verified the successful transformation from $\mathrm{Fe}_{3} \mathrm{O}_{4}$ NPs to PB NPs, which was carried in the immunosensing solution in the presence of the analyte. In addition, quantitative colorimetric detection of PSA was performed based on UV-Vis absorbance using the microplate reader. Fig. 2B displays the calibration curve (blue) between absorbances and concentrations of the target analyte PSA analyte. The results turned out that the absorbances at $748 \mathrm{~nm}$ were proportional to the logarithmical concentrations of PSA spiked in the PBS buffer. A linear relationship was established with the $\mathrm{R}^{2}$ value of 0.988 . The LOD of this colorimetric assay using a microplate reader was calculated to be as low as $1.0 \mathrm{ng} / \mathrm{mL}$ based on 3 -fold standard deviations above the blank signal, which was comparable to the sensitivity of commercial PSA ELISA kits (LOD: 1.0 $\mathrm{ng} / \mathrm{mL}) .{ }^{47}$ Moreover, the good linearity between absorbances in the NIR range (i.e., at $810 \mathrm{~nm}$ ) and the logarithmical concentrations of PSA was obtained in Fig. 2B (red), which further provided a solid foundation for the following quantitative and sensitive detection of biomarkers based on the photothermal effect-driven principle.
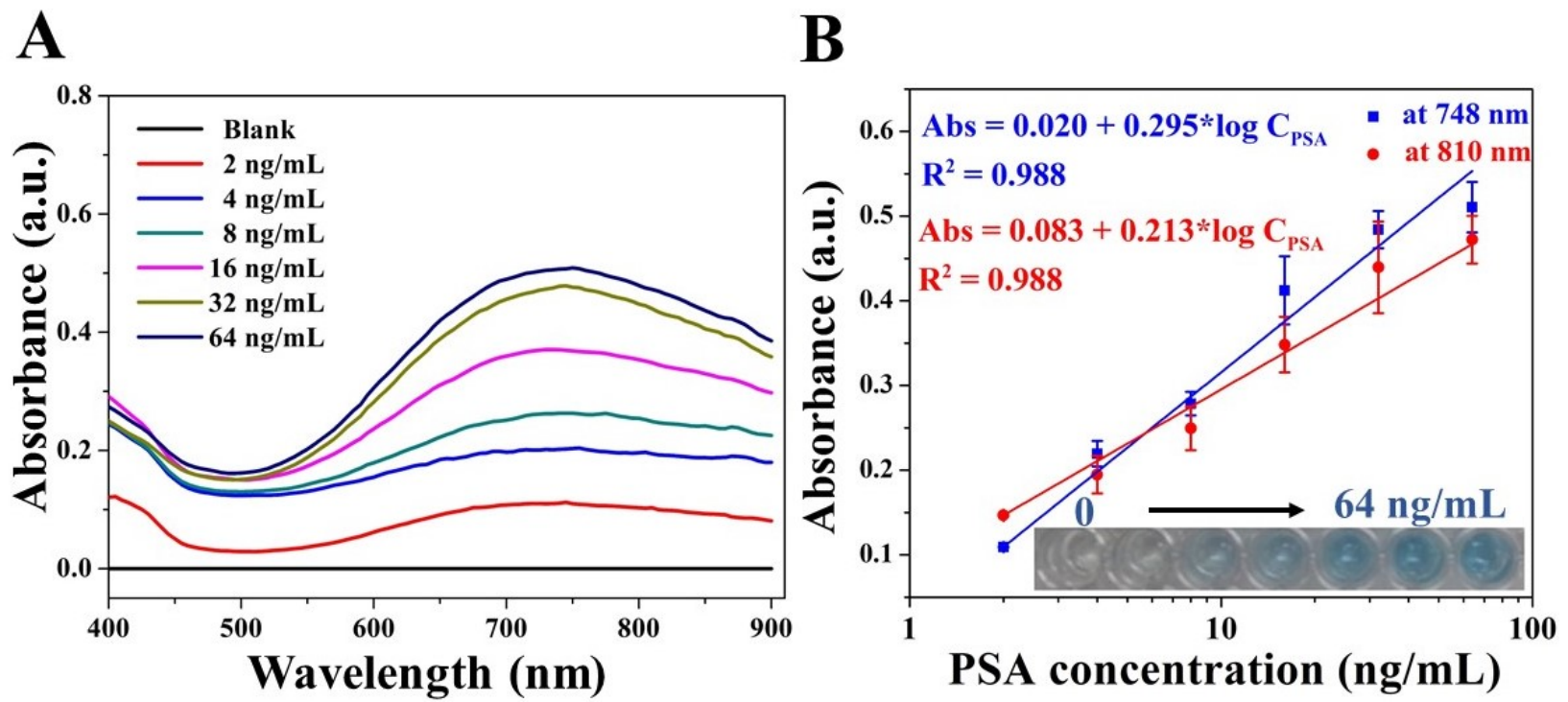

Fig. 2 UV-vis Characterization of PSA detection in the PBS buffer. (A) UV-vis spectra of immunosensing solutions after the transformation from $\mathrm{Fe}_{3} \mathrm{O}_{4}$ NPs to PB NPs at different PSA concentrations (0-64 ng/mL) prepared in the PBS buffer. (B) Calibration plots of absorbance at $748 \mathrm{~nm}$ and $810 \mathrm{~nm}$ vs. the logarithm of the PSA concentration. Insets are the photographs of the above immunosensing solutions. Error bars represent standard deviations $(n=3)$. 


\section{Visual quantitative detection of PSA in PBS using the PDMS/PMMA hybrid PT-Chip}

After the UV-vis absorbance characterization of the PSA immunosensing system, we performed visual quantitative detection of various concentrations of PSA in PBS using the PDMS/PMMA hybrid PT-Chip. Once the immunosensing solutions were loaded to the central microwells on the PDMS/PMMA hybrid PT-Chip, four different samples were irradiated by the $808 \mathrm{~nm}$ laser at the same time. With one-time irradiation at the power density of $2.21 \mathrm{~W} / \mathrm{cm}^{2}$, all sample solutions started to flow through microchannels due to the photothermal effect-driven principle (Fig. 3). The fluid movement could be easily observed by naked-eyes, and the moving distance $(\Delta \mathrm{L})$ through the microchannels was recorded as the readout according to the designed on-chip scale ruler immediately after the 5 min irradiation. It is noted that the yellow-colored background was used for enhanced visual contrast between the background and the colored bar charts in microfluidic channels. As shown in Fig. 3A, with the increase of PSA concentrations, the moving distances of sample solutions were clearly elongated under the laser irradiation. In the control tests $(0 \mathrm{ng} / \mathrm{mL}$ PSA), the $\Delta \mathrm{L}$ value was acquired to be minimal (0.54 a.u.), whereas a $\Delta \mathrm{L}$ of 10.45 a.u. was observed at the PSA concentration of $64 \mathrm{ng} / \mathrm{mL}$ (Note: 1 a.u. $=2.0 \mathrm{~mm}$ ). A linear relationship (Fig. 3B) was obtained between the moving distances and the logarithmical concentrations of PSA in the range from 2.0 to $64.0 \mathrm{ng} / \mathrm{mL}$, with the $\mathrm{R}^{2}$ value of 0.986 . The LOD was determined to be 2.0 ng/mL. Although no bulky and costly instruments were used, our PT-Chip demonstrated similar detection sensitivity to the colorimetric method using a microplate reader (LOD, $1.0 \mathrm{ng} / \mathrm{mL}$ ), showing great potential for clinical diagnostics with the cut-off PSA concentration of $4.0 \mathrm{ng} / \mathrm{mL}$. This series of studies also built a solid framework for us to move forward to test more complicated human samples. 

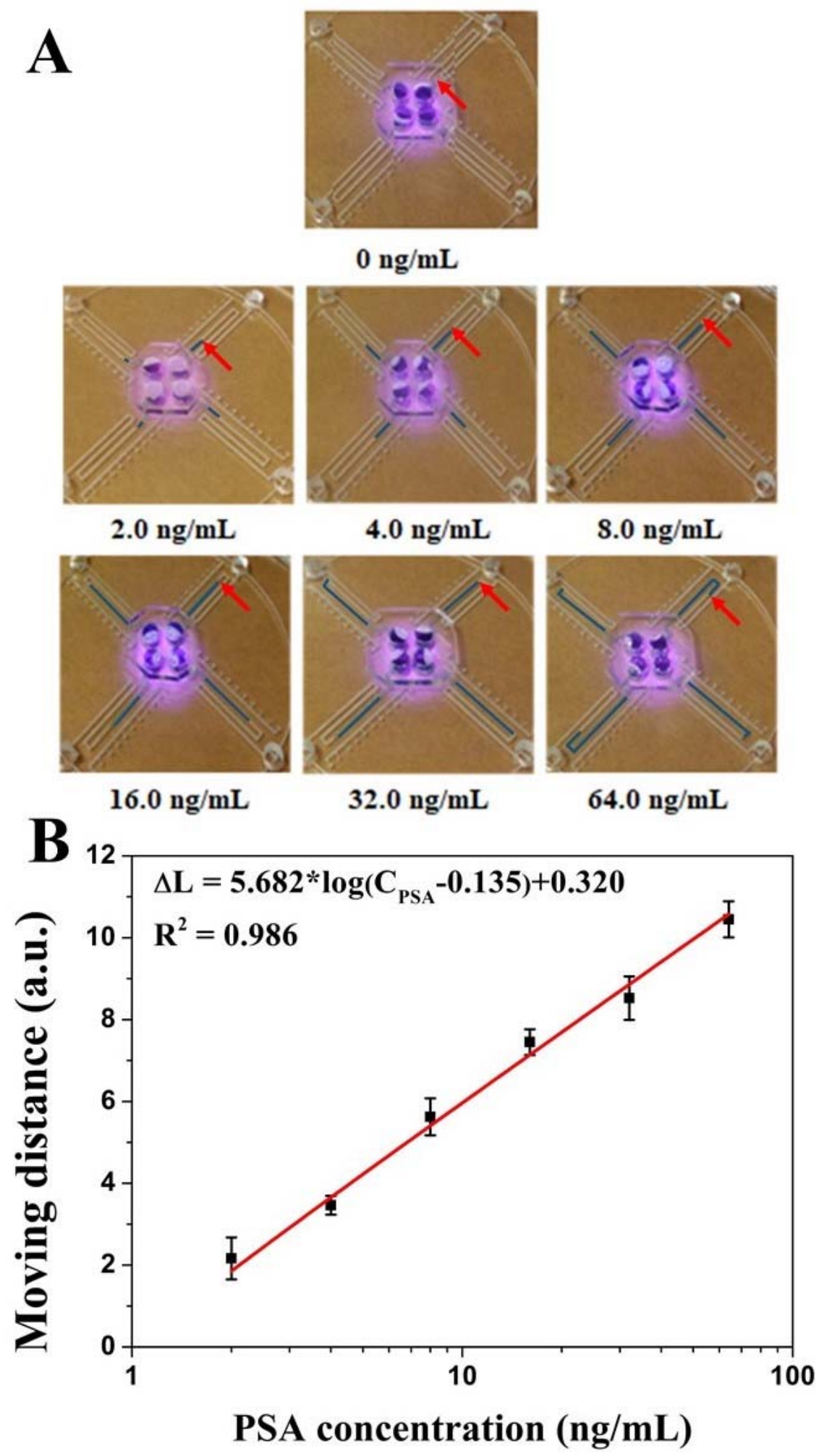

Fig. 3 Visual quantitative detection of PSA in PBS using the PDMS/PMMA hybrid PT-Chip. (A) Photographs of on-chip visual detection of different concentrations of PSA PBS. Four parallel 
experiments were conducted simultaneously on a single chip. Red arrows indicate the end location of bar-chart movements. (B) Calibration plot of moving distances ( $\Delta$ Ls) vs. the logarithmical concentrations of PSA. Error bars represent standard deviations $(n=4) .1$ a.u. $=2.0 \mathrm{~mm}$. The laser irradiation time was $5 \mathrm{~min}$ at the laser power density of $2.21 \mathrm{~W} / \mathrm{cm}^{2}$.

\section{Colorimetric immunosensing of PSA in human serum samples using a microplate reader}

Human serum is the standard sample used in current clinical diagnostic testing of PSA. ${ }^{48-50}$ To validate our method and examine the matrix effects, normal human serum samples spiked with different concentrations of PSA were employed instead of PBS buffers. Similarly, we first tested the feasibility by using colorimetric detection of PSA in human serum samples using a microplate reader before the on-chip photothermal bar-chart detection. UV-vis characterization of the colorimetric ELISA of PSA in human serum was performed using a microplate reader, and the results are shown in Fig. 4. As the PSA concentration increased, an obvious and enhanced blue color appeared in the sample solutions (Fig. 4A), confirming the successful immunorecognition and the subsequent nanoparticle transformation. From the UV-vis spectra (Fig. 4B), a calibration curve was obtained between the absorbance at $748 \mathrm{~nm}$ and the logarithms of the PSA concentrations (Fig. 4C), which were linearly correlated, with an R-squared value of 0.98 . The LOD of $1.3 \mathrm{ng} / \mathrm{mL}$ PSA was obtained in serum samples, which was similar to the LOD $(1.0 \mathrm{ng} / \mathrm{mL})$ obtained in PBS buffers, indicating the feasibility for our subsequent PT-Chip immunosensing of human samples. 


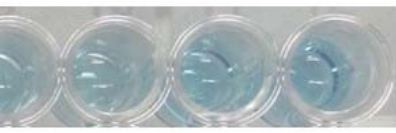

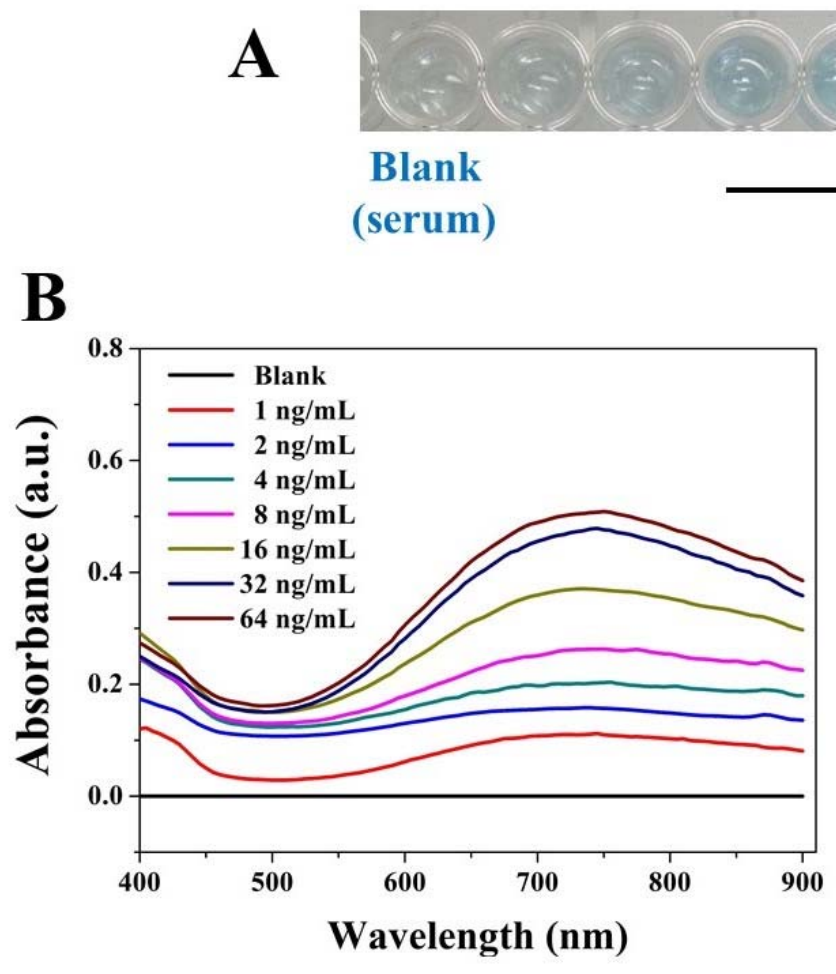

Blank

(serum)
$64 \mathrm{ng} / \mathrm{mL}$

(PSA concentration)

C

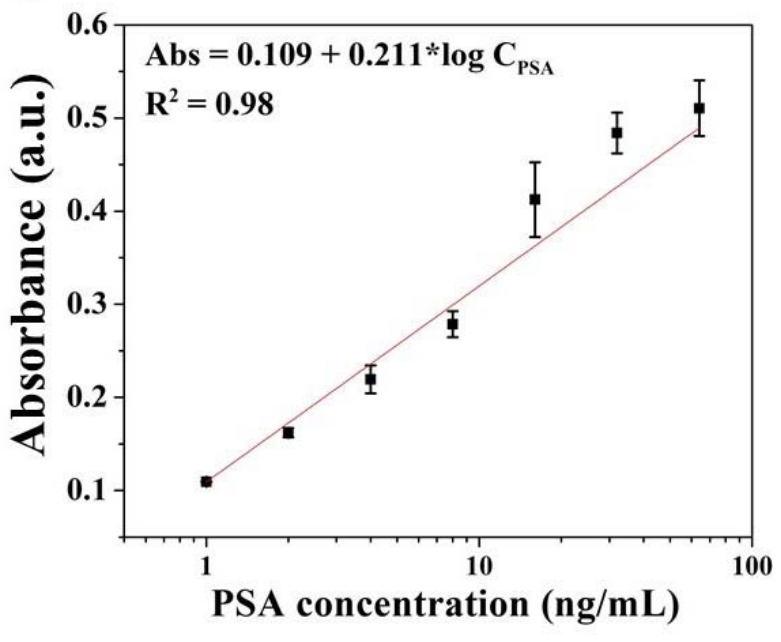

Fig. 4 UV-vis characterization of PSA detection in serum samples. (A) Photographs and (B) UVvis spectra of immunosensing solutions after the transformation from $\mathrm{Fe}_{3} \mathrm{O}_{4}$ NPs to PB NPs at different PSA concentrations (0-64 ng/mL) spiked in serum samples. (C) Calibration plot of absorbance at $748 \mathrm{~nm}$ vs. logarithm of the PSA concentration. Error bars represent standard deviations $(\mathrm{n}=3)$.

Visual quantitative detection of PSA in human serum samples using the PDMS/PMMA hybrid PT-Chip

After demonstrating the feasibility of using UV-vis absorbance, we explored the visual quantitative detection of PSA in human serum samples using the PDMS/PMMA hybrid PT-Chip. In the onchip detection, under the NIR laser irradiation, the moving distance $(\Delta \mathrm{L})$ of varying concentrations of PSA spiked in normal serum samples was recorded. The results in Fig. 5A show that the moving distance of sample solutions was prolonged on the PT-Chip as the PSA concentrations increased. The moving distance was proportional to the concentration of PSA in spiked serum samples upon laser irradiation for $5 \mathrm{~min}$. There was a linear relationship between $\Delta \mathrm{L}$ and the logarithmical concentration of PSA in the range from 1.0 to $64.0 \mathrm{ng} / \mathrm{mL}$, with the coefficient of determination as 0.986 in Fig. 5B. The LOD was calculated to be $2.1 \mathrm{ng} / \mathrm{mL}$, which was below the clinical 
threshold value of $4.0 \mathrm{ng} / \mathrm{mL}$. This LOD is similar to that of the on-chip PSA testing in PBS (2.0 $\mathrm{ng} / \mathrm{mL})$ as well as the LOD value from the absorbance measurement of serum samples $(1.3 \mathrm{ng} / \mathrm{mL})$. The results confirmed that visual quantitative detection of PSA can be achieved using the PT-Chip, even in complex media such as human serum samples without compromising the detection sensitivity, indicating the robustness of our immunosensing method.

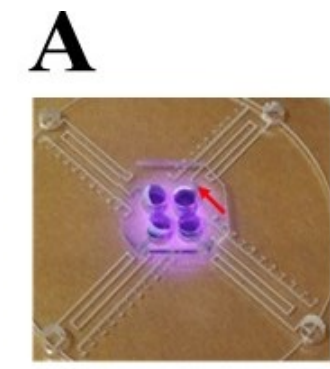

0 ng/mL

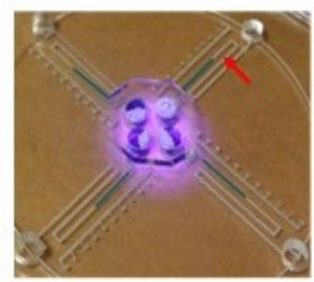

$8 \mathrm{ng} / \mathrm{mL}$

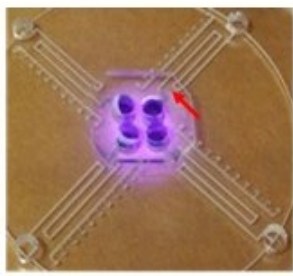

$1 \mathrm{ng} / \mathrm{mL}$

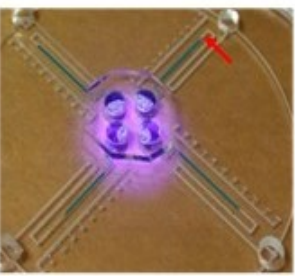

$16 \mathrm{ng} / \mathrm{mL}$

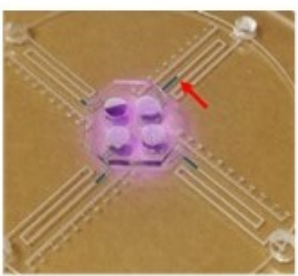

$2 \mathrm{ng} / \mathrm{mL}$

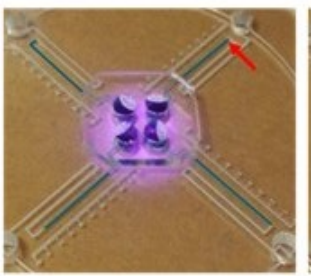

$32 \mathrm{ng} / \mathrm{mL}$

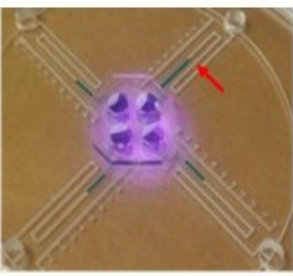

$4 \mathrm{ng} / \mathrm{mL}$

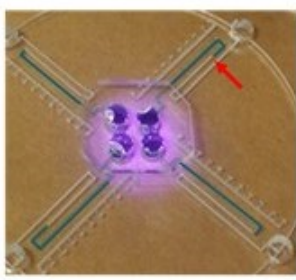

$64 \mathrm{ng} / \mathrm{mL}$

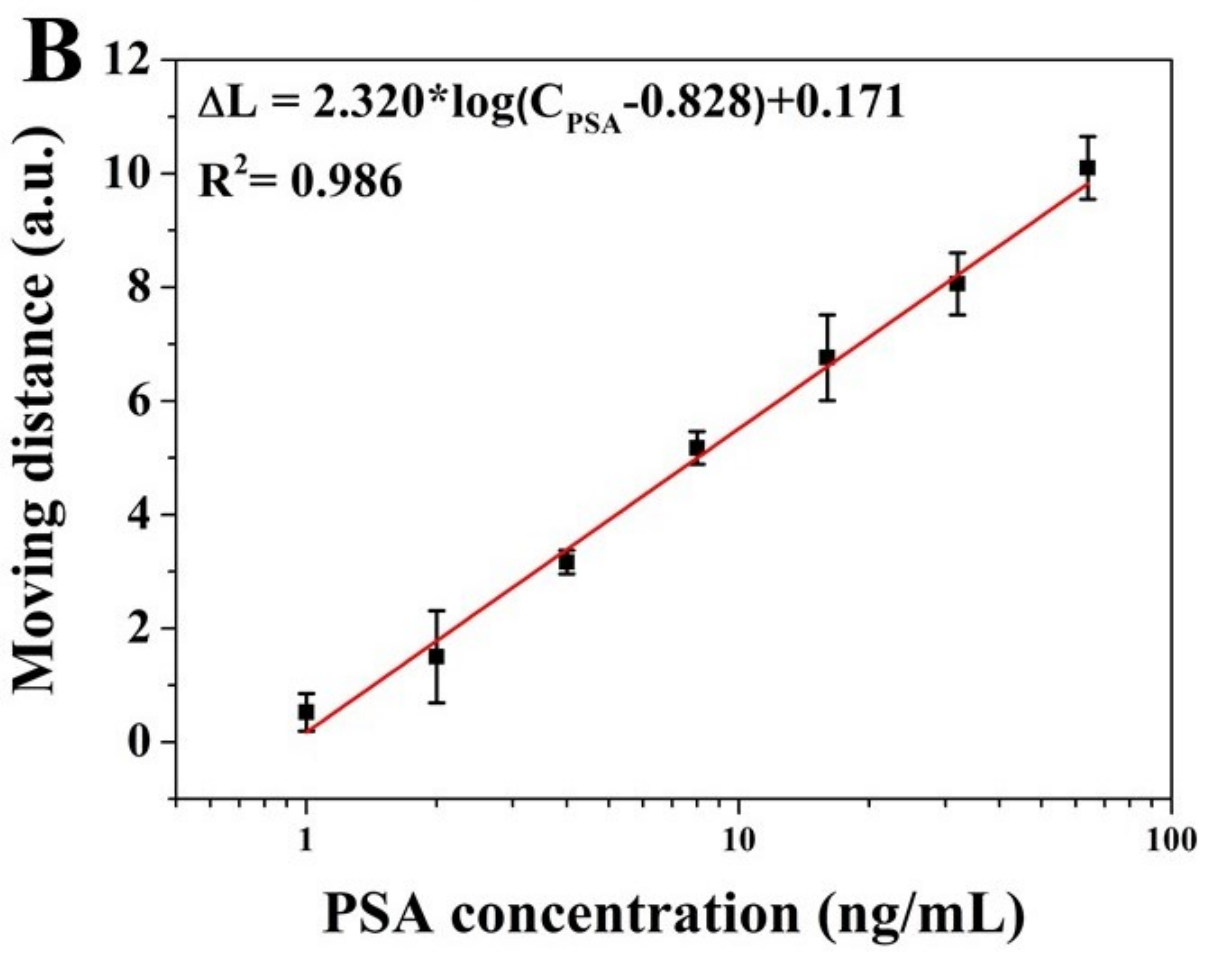


Fig. 5 Visual quantitative detection of PSA spiked in human serum samples using the PDMS/PMMA hybrid PT-Chip. (A) Photographs of on-chip detection of different concentrations of PSA spiked in human serum samples. Four parallel experiments were conducted on a single chip at the same time. Red arrows indicate the stop point of bar charts. (B) Calibration plot of moving distance $(\Delta \mathrm{Ls})$ vs. the logarithmical concentrations of spiking PSA. Error bars represent standard deviations $(\mathrm{n}=4) .1$ a.u. $=2.0 \mathrm{~mm}$. The laser irradiation time was $5 \mathrm{~min}$ at the laser power density of $2.21 \mathrm{~W} / \mathrm{cm}^{2}$.

\section{Specificity tests and analytical recoveries}

To study the specificity of the photothermal bar-chart chip method, some common interfering substances, including serum, human whole blood, HBsAg, CEA, and IgG with higher concentrations up to $80 \mathrm{ng} / \mathrm{mL}$, were prepared and tested using a similar procedure on the PT-Chip. As shown in Fig. 6, compared with blank samples containing only serum or blood matrix media, only samples spiked with PSA had obvious moving distance (in the range of 3-6 a.u.), whereas no significant bar-chart movements were observed from other interfering substances, indicating high specificity of our PT-Chip method.

To further evaluate the analytical performance of the photothermal bar-chart chip method in complex media, we also compared the PT-Chip testing results between human serum and human whole blood samples spiked with different concentrations of PSA. Two concentrations of PSA, 4 $\mathrm{ng} / \mathrm{mL}$ and $8 \mathrm{ng} / \mathrm{mL}$, were selected in this comparison according to the commonly used threshold concentration and the suspect level for clinical prostate cancer diagnostics. ${ }^{51-53}$ As shown in Fig. 6, $4 \mathrm{ng} / \mathrm{mL}$ PSA gave bar-chart values of 3.17 and 3.08 a.u. from serum and whole blood, respectively; while $8 \mathrm{ng} / \mathrm{mL}$ PSA gave bar-chart values of 5.18 and 5.04 a.u. from serum and whole blood, respectively. Besides, the $t$-test statistics further confirmed the results between serum and whole blood were similar, with $\mathrm{P}$ values of 0.58 and 0.48 ( $\mathrm{P}$-value $>0.05$ ). The results demonstrated high tolerance of our visual quantitative PT-Chip method to matrix effects from complex media. Furthermore, no color interferences were observed in the whole blood tests using our PT-Chip. Given that current diagnostic methods require the use of serum due to the color interference problem from red blood cells, our visual photothermal bar-chart method is capable of testing whole blood directly, which may fill a gap in the current clinical diagnostics paradigm. 


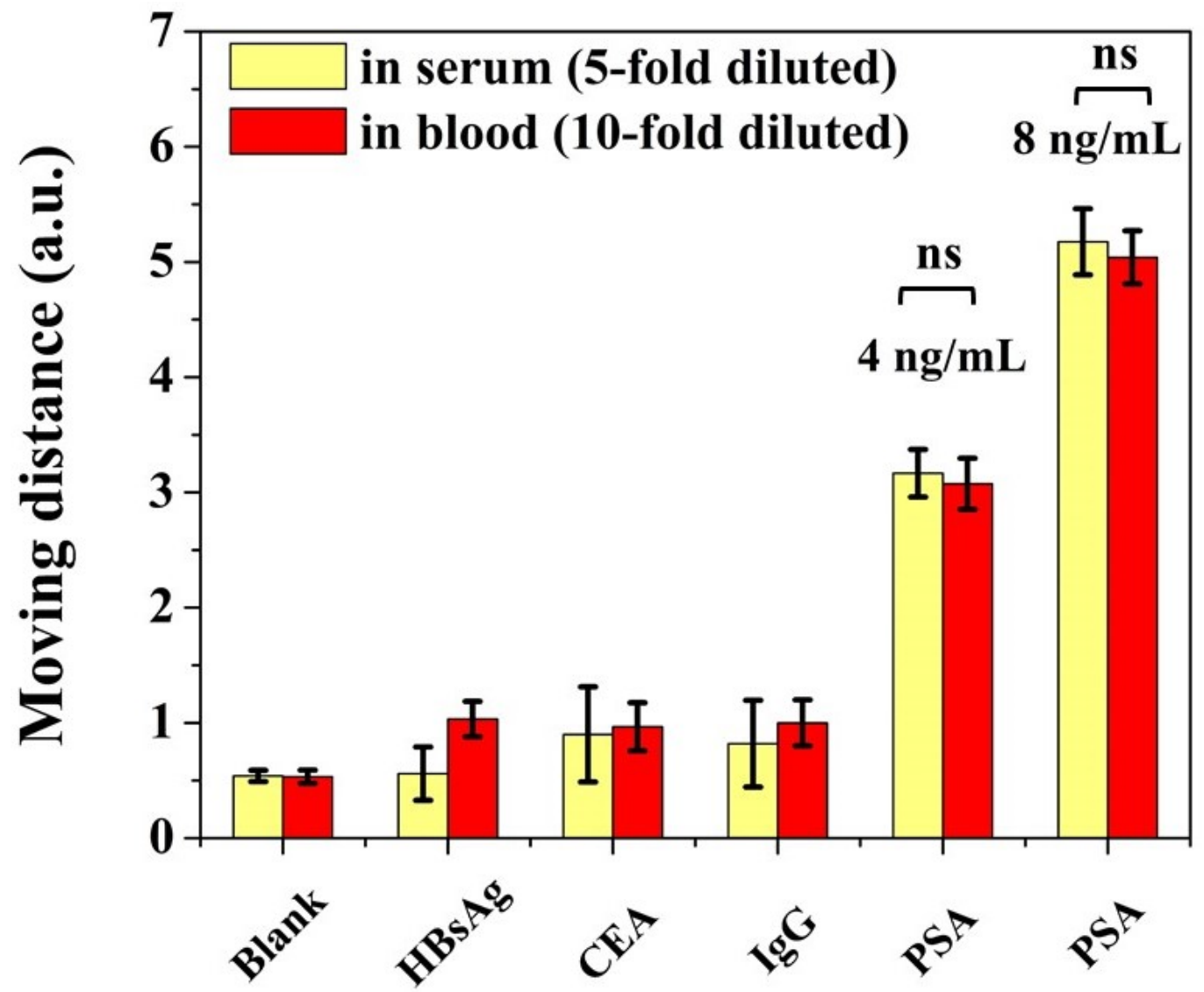

Fig. 6 Specificity tests for PSA detection using the PT-Chip in different matrix media, including 5-fold diluted normal human serum and 10-fold diluted human whole blood samples. Other interfering substances were tested including $\mathrm{HBsAg}$, CEA, and IgG with a spiked concentration of $80 \mathrm{ng} / \mathrm{mL}$. The media (serum and whole blood) were used as the blanks. Error bars represent standard deviations $(\mathrm{n}=4)$. The symbol $(\mathrm{ns})$ indicates no statistical significance in the $t$-test $(\mathrm{P}-$ value $>0.05)$.

Furthermore, our PT-Chip method was validated by testing the analytical recoveries of PSA spiked in serum and human whole blood samples. Similarly, we chose two PSA concentrations at the cutoff level and the suspect level in both matrix samples (i.e., 4 and $8 \mathrm{ng} / \mathrm{mL}$, respectively). By comparing the known spiked concentrations in the immunoassay and the measured values of PSA using the PT-Chip, the analytical recoveries were calculated and summarized in Table 1. It was found that the analytical recoveries with PSA concentrations of 4 and $8 \mathrm{ng} / \mathrm{mL}$ in human serum were $91.3 \%$ to $91.9 \%$, respectively. The analytical recoveries obtained in spiked human whole blood samples were $92.5 \%$ to $89.1 \%$, respectively. Their RSD values ranged from $4.5 \%$ to $7.0 \%$. 
Overall, all the analytical recoveries were within the acceptable criteria for bioanalytical method validation, ${ }^{54,55}$ indicating that our PT-Chip yields reliable results with satisfactory interpretation for the detection of PSA even in the human whole blood matrix.

Table 1 Analytical recoveries for the detection of PSA spiked in normal human serum samples and human whole blood samples

\begin{tabular}{cccccc}
\hline Matrix & $\begin{array}{c}\text { Sample } \\
\text { No. }\end{array}$ & $\begin{array}{c}\text { PSA spiking } \\
\text { concentration } \\
(\mathrm{ng} / \mathrm{mL})\end{array}$ & $\begin{array}{c}\text { PSA detected } \\
\text { concentration } \\
(\mathrm{ng} / \mathrm{mL})\end{array}$ & Recovery (\%) & RSD (\%) \\
\hline $\begin{array}{c}\text { Normal } \\
\text { human serum } \\
(\mathrm{n}=4)\end{array}$ & 1 & 4.0 & 3.65 & 91.3 & 5.9 \\
\hline $\begin{array}{c}\text { Human whole } \\
\text { blood (n=4) }\end{array}$ & 3 & 8.0 & 7.35 & 91.9 & 5.1 \\
\hline
\end{tabular}

\section{Conclusion}

In conclusion, photothermal effects as a new type of driving force have been for the first time applied to V-Chip, and thus an innovative photothermal bar-chart microfluidic chip (PT-Chip) has been developed for visual quantitative detection of biochemicals at the point of care, without the use of any costly and bulky instrument. PSA as a model analyte was successfully detected using this PT-Chip from different types of matrices including PBS buffers, human serum, and human whole blood samples, with high sensitivity and specificity. The LOD of $2.1 \mathrm{ng} / \mathrm{mL}$ PSA was obtained in normal human serum samples, which met the cut-off requirement in clinical diagnostics for prostate cancers. This detection platform was further validated by testing spiked human whole blood samples, obtaining satisfactory analytical recoveries, demonstrating good analytical performance for biomarker detection even in the complex matrix.

This new type of V-Chip, PT-Chip, has many significant features. (1) It is miniaturized, portable, and does not require any costly and bulky conventional analytical instruments, enhancing the capability for POC detection in various settings, especially those resource-poor settings, such as households, small clinics, rural areas, and developing nations. (2) The produced signals (i.e., onchip bar-chart movements) are easy to visualize, read, and record, with no need for trained personnel and complex data analytics. (3) Compared to the conventional gas-driven V-Chips based 
on chemical reactions, the PT-Chip using nanomaterial-mediated photothermal effects as the driving force has precise controllability in controlling reaction progresses and the location of the reactions on the chip, which can be remotely tuned by adjusting parameters of laser irradiation. Relevant work with high degrees of integration is underway in our laboratory. (4) Given the nature of the readout based on bar-chart movement instead of color changes, the PT-Chip has unique advantages in quantitative POC detection of colored samples without interference problems. As demonstrated in this work, PSA was successfully detected in human whole blood, without noticeable differences from serum testing results. Because current diagnostic methods require precentrifugation to obtain serum before biomarker assays, our PT-Chip capable of whole blood testing can fill a gap in the conventional clinical diagnostics paradigm. (5) The fabrication of PTChip is simple and low cost due to the use of inexpensive materials (i.e., PDMS and PMMA), as compared with glass-based V-Chips that require cleanroom and complex and costly fabrication procedures. No specialized infrastructure (like cleanroom) and complicated assembly processes are needed. (6) Multiple microwells from the PT-Chip allow the versatile and multiplexed detection of multiple analytes/samples simultaneously. Although only 4 analytical units are designed in our current PT-Chip, more analytical units can be scaled up. Overall, by combining nanomaterial-mediated photothermal effects with microfluidic LOC devices, the PT-Chip has great potential in the exploration of new POC diagnostic tools for broad applications, particularly given more inexpensive, portable, and powerful laser pointers becoming commercially available recently. The novel introduction of nanomaterial-mediated photothermal effects into bar-chart microfluidic chips opens a new era towards advances in clinical diagnostics and POC analysis, as well as for the exploration of new application fields of photothermal effects.

\section{Acknowledgments}

We would like to acknowledge the financial support from the National Institute of Allergy and Infectious Disease of the NIH (R21AI107415), the U.S. NSF (IIP2052347, IIP1953841), DOT (CARTEEH), the University of Texas at El Paso (UTEP) for the IDR Program, the Philadelphia Foundation, and the Medical Center of the Americas Foundation. We are also grateful for the financial support to our prior research from the National Institute of General Medical Sciences of the NIH (SC2GM105584), the NIH RCMI Pilot grant, the NIH BUILDing Scholar Summer Sabbatical Award, NSF (DMR1827745), University of Texas (UT) System for the STARS award, 
and the University of Texas at El Paso (UTEP) for the Multidisciplinary Research Award Program (MRAP) and URI Program. 


\section{References}

1. K. Strimbu and J. A. Tavel, Curr. Opin. HIV AIDS, 2010, 5, 463-466.

2. A. Ganna, S. Salihovic, J. Sundstrom, C. D. Broeckling, A. K. Hedman, P. K. E. Magnusson, N. L. Pedersen, A. Larsson, A. Siegbahn, M. Zilmer, J. Prenni, J. Arnlov, L. Lind, T. Fall and E. Ingelsson, PLoS Genet., 2014, 10, e1004801.

3. S. N. Topkaya, M. Azimzadeh and M. Ozsoz, Electroanalysis, 2016, 28, 1402-1419.

4. F. M. Zou, H. J. Zhou, T. V. Tan, J. Kim, K. Koh and J. Lee, ACS Appl. Mater. Interfaces, 2015, 7, 12168-12175.

5. F. L. Qu, T. Li and M. H. Yang, Biosens. Bioelectron., 2011, 26, 3927-3931.

6. G. Fu, S. T. Sanjay, W. Zhou, R. A. Brekken, R. A. Kirken and X. Li, Anal. Chem., 2018, 90, 5930-5937.

7. A. B. Chinen, C. M. Guan, J. R. Ferrer, S. N. Barnaby, T. J. Merkel and C. A. Mirkin, Chem. Rev., 2015, 115, 10530-10574.

8. B. V. Chikkaveeraiah, A. A. Bhirde, N. Y. Morgan, H. S. Eden and X. Chen, ACS Nano, 2012, 6, 65466561.

9. $\quad$ S. Bi, H. Zhou and S. S. Zhang, Biosens. Bioelectron., 2009, 24, 2961-2966.

10. S. T. Sanjay, G. L. Fu, M. W. Dou, F. Xu, R. T. Liu, H. Qi and X. J. Li, Analyst, 2015, 140, 7062-7081.

11. A. V. Ritchie, N. Goel, H. Sembongi, J. Lehga, L. E. Farleigh, D. Edemaga, C. A. Wisniewski and H. H. Lee, J. Virol. Methods, 2016, 237, 143-149.

12. N. A. Schultz, C. Dehlendorff, B. V. Jensen, J. K. Bjerregaard, K. R. Nielsen, S. E. Bojesen, D. Calatayud, S. E. Nielsen, M. Yilmaz, N. H. Hollander, K. K. Andersen and J. S. Johansen, Jama, 2014, 311, 392-404.

13. M. Lv, W. Zhou, H. Tavakoli, C. Bautista, J. Xia, Z. Wang and X. Li, Biosens. Bioelectron., 2021, 176, 112947.

14. M. W. Dou, S. T. Sanjay, M. Benhabib, F. Xu and X. J. Li, Talanta, 2015, 145, 43-54.

15. W. Zhou, M. Feng, A. Valadez and X. Li, Anal. Chem., 2020, 92, 7045-7053.

16. K. S. Prasad, X. Cao, N. Gao, Q. Jin, S. T. Sanjay, G. Henao-Pabon and X. Li, Sensors Actuators B: Chem., 2020, 305, 127516.

17. H. Tavakoli, W. Zhou, L. Ma, S. Perez, A. Ibarra, F. Xu, S. Zhan and X. Li, TrAC, Trends Anal. Chem., 2019.

18. H. Tavakoli, W. Zhou, L. Ma, Q. Guo and X. Li, Nanotechnology and Microfluidics, 2020, 177-209.

19. X. Wei, W. Zhou, S. T. Sanjay, J. Zhang, Q. Jin, F. Xu, D. C. Dominguez and X. Li, Anal. Chem., 2018, 90, 9888-9896.

20. S. T. Sanjay, W. Zhou, M. W. Dou, H. Tavakoli, L. Ma, F. Xu and X. J. Li, Adv. Drug Deliv. Rev., 2018, 128, 3-28.

21. Y. Song, Y. Zhang, P. E. Bernard, J. M. Reuben, N. T. Ueno, R. B. Arlinghaus, Y. Zu and L. Qin, Nat. Commun., 2012, 3, 1283.

22. Y. J. Song, X. F. Xia, X. F. Wu, P. Wang and L. D. Qin, Angew. Chem. Int. Ed., 2014, 53, 12451-12455.

23. Y. Song, Y. Li and L. Qin, Methods Mol. Biol., 2017, 1570, 105-115.

24. Z. Zhu, Z. C. Guan, S. S. Jia, Z. C. Lei, S. C. Lin, H. M. Zhang, Y. L. Ma, Z. Q. Tian and C. J. Yang, Angew. Chem. Int. Ed., 2014, 53, 12503-12507.

25. Y. Z. Wang, G. X. Zhu, W. J. Qi, Y. Li and Y. J. Song, Biosens. Bioelectron., 2016, 85, 777-784.

26. Y. J. Song, Y. Z. Wang, W. J. Qi, Y. Li, J. Xuan, P. Wang and L. D. Qin, Lab Chip, 2016, 16, 2955-2962.

27. Y. Li, J. Xuan, T. Xia, X. Han, Y. J. Song, Z. Cao, X. Jiang, Y. Guo, P. Wang and L. D. Qin, Anal. Chem., 2015, 87, 3771-3777.

28. G. Fu, Y. Zhu, W. Wang, M. Zhou and X. Li, ACS Sensors, 2019, 4, 2481-2490.

29. G. L. Fu, Y. B. Zhu, K. Xu, W. H. Wang, R. X. Hou and X. J. Li, Anal. Chem., 2019, 91, 13290-13296. 
30. G. L. Fu, S. T. Sanjay, W. Zhou, R. A. Brekken, R. A. Kirken and X. J. Li, Anal. Chem., 2018, 90, 59305937.

31. G. L. Hong, D. D. Zhang, Y. H. He, Y. Y. Yang, P. Chen, H. J. Yang, Z. Y. Zhou, Y. H. Liu and Y. Wang, Anal. Bioanal. Chem., 2019, 411, 6837-6845.

32. S. Y. Du, Y. Wang, Z. C. Liu, Z. X. Xu and H. Y. Zhang, Biosens. Bioelectron., 2019, 144, 111670.

33. G. L. Fu, S. T. Sanjay and X. J. Li, Analyst, 2016, 141, 3883-3889.

34. G. Fu, S. T. Sanjay, M. Dou and X. Li, Nanoscale, 2016, 8, 5422-5427.

35. J. Zhang, H. Xing and Y. Lu, Chemical Science, 2018, 9, 3906-3910.

36. W. Zhou, K. Q. Hu, S. Kwee, L. Tang, Z. H. Wang, J. F. Xia and X. J. Li, Anal. Chem., 2020, 92, 27392747.

37. W. Zhou, J. Sun and X. Li, Anal. Chem., 2020, 92, 14830-14837.

38. G. Fu, W. Zhou and X. Li, Lab Chip, 2020, 20, 2218-2227.

39. D. A. Hastman, J. S. Melinger, G. L. Aragones, P. D. Cunningham, M. Chiriboga, Z. J. Salvato, T. M. Salvato, C. W. Brown, 3rd, D. Mathur, I. L. Medintz, E. Oh and S. A. Diaz, ACS Nano, 2020, 14, 85708583.

40. Y. Liu, M. Pan, W. Wang, Q. Jiang, F. Wang, D. W. Pang and X. Liu, Anal. Chem., 2019, 91, 20862092.

41. L. Huang, J. Chen, Z. Yu and D. Tang, Anal. Chem., 2020, 92, 2809-2814.

42. M. W. Dou, S. T. Sanjay, D. C. Dominguez, P. Liu, F. Xu and X. J. Li, Biosens. Bioelectron., 2017, 87, 865-873.

43. R. W. R. L. Gajasinghe, S. U. Senveli, S. Rawal, A. Williams, A. Zheng, R. H. Datar, R. J. Cote and O. Tigli, J. Micromech. Microeng., 2014, 24, 075010.

44. T. Waritani, J. Chang, B. McKinney and K. Terato, MethodsX, 2017, 4, 153-165.

45. G. Fu, S. T. Sanjay and X. Li, Analyst, 2016, 141, 3883-3889.

46. G. Fu, W. Liu, S. Feng and X. Yue, Chem. Commun., 2012, 48, 11567-11569.

47. Z. Gao, L. Hou, M. Xu and D. Tang, Sci. Rep., 2014, 4, 3966.

48. H. Chen, Z. Li, L. Zhang, P. Sawaya, J. Shi and P. Wang, Angew. Chem., 2019, 131, 14060-14066.

49. N. J. Sathianathen, B. R. Konety, J. Crook, F. Saad and N. Lawrentschuk, Nat. Rev. Urol., 2018, 15, 627-642.

50. L. Yang, J. Zheng, Z. Zou, H. Cai, P. Qi, Z. Qing, Q. Yan, L. Qiu, W. Tan and R. Yang, Chem. Commun., 2020, 56, 1843-1846.

51. G. Bartsch, Urology, 2002, 59, 893-894.

52. A. G. Anastasiadis, M. P. Lichy, U. Nagele, M. A. Kuczyk, A. S. Merseburger, J. Hennenlotter, S. Corvin, K. D. Sievert, C. D. Claussen, A. Stenzl and H. P. Schlemmer, Eur. Urol., 2006, 50, 738-749.

53. F. Aigner, L. Pallwein, D. Junker, G. Schäfer, G. Mikuz, F. Pedross, M. J. Mitterberger, W. Jaschke, E. J. Halpern and F. Frauscher, J. Urol., 2010, 184, 913-917.

54. S. Zhou, W. Zheng, Z. Chen, D. Tu, Y. Liu, E. Ma, R. Li, H. Zhu, M. Huang and X. Chen, Angew. Chem. Int. Ed., 2014, 53, 12498-12502.

55. G. Tiwari and R. Tiwari, Pharm. Methods, 2010, 1, 25-38. 


\title{
Supporting Information
}

\section{Detector-free photothermal bar-chart microfluidic chips (PT-Chips) for visual quantitative detection of biomarkers}

\author{
Wan Zhou, ${ }^{1+}$ Guanglei $\mathrm{Fu}^{1,2+}$ and Xiujun $\mathrm{Li}^{1,3,4^{*}}$ \\ ${ }^{1}$ Department of Chemistry and Biochemistry, The University of Texas at El Paso, 500 West \\ University Ave, El Paso, TX 79968, USA \\ 2Biomedical Engineering Research Center, Medical School of Ningbo University, Ningbo, \\ Zhejiang, 315211, P. R. China \\ ${ }^{3}$ Border Biomedical Research Center, Biomedical Engineering, The University of Texas at El \\ Paso, 500 West University Ave, El Paso, TX 79968, USA \\ ${ }^{4}$ Environmental Science and Engineering, The University of Texas at El Paso, 500 West \\ University Ave, El Paso, TX 79968, US \\ ${ }^{+}$Denotes equal contributions. \\ * Corresponding Author: XiuJun Li; Email: xli4@utep.edu
}


Figure S1.

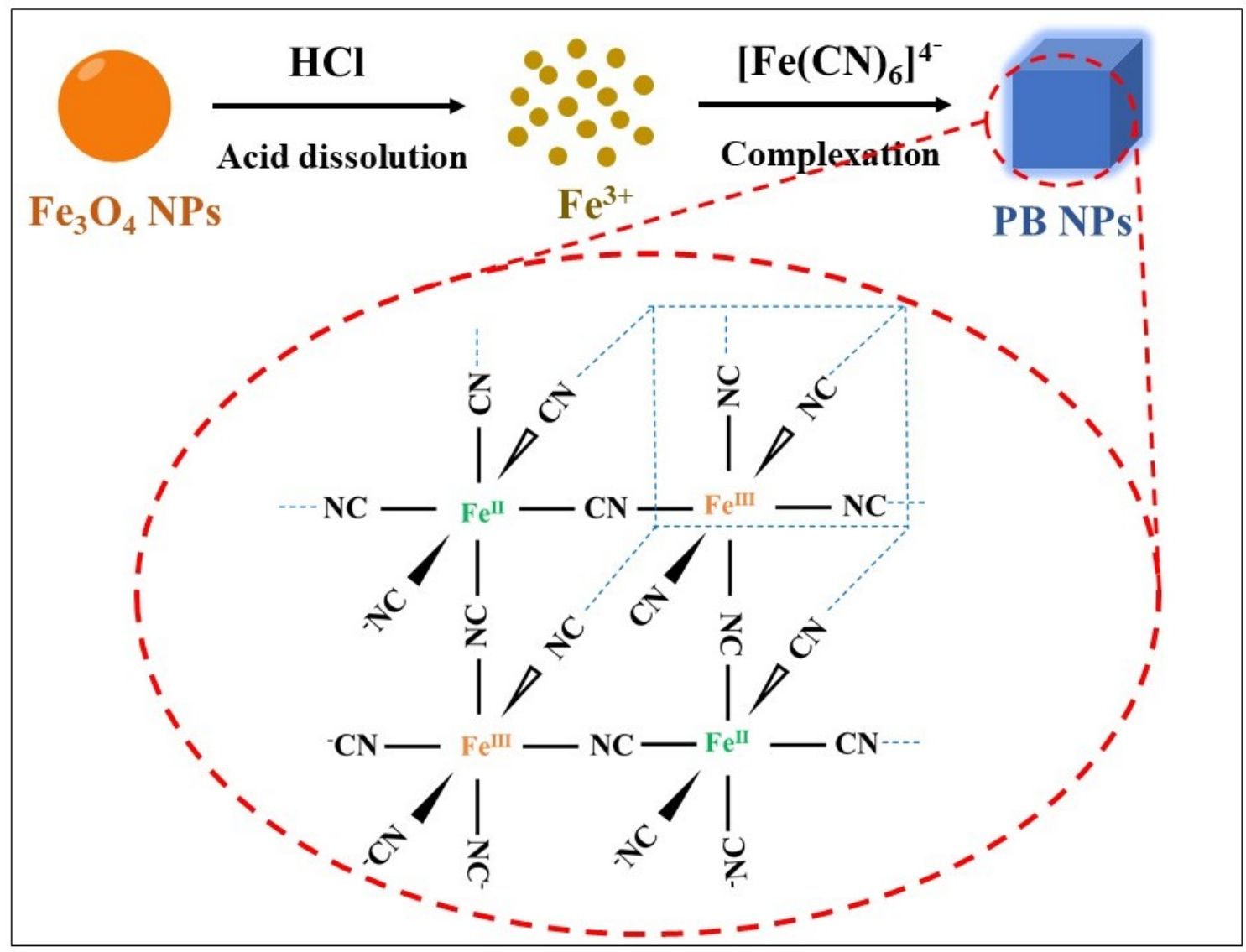

Figure S1. The transformation reaction from $\mathrm{Fe}_{3} \mathrm{O}_{4}$ NPs to PB NPs via complexation reactions. 